\title{
Anti-diabetic Effects of Ethanol Extract from Bitter Melon in Mice Fed a High-fat Diet
}

\author{
Nal Ae Yoon', Juyeong Park ${ }^{1}$, Jiyeon Lee ${ }^{1}$, Joo Yeon Jeong ${ }^{1}$, Hyun-Kyu Kim² , Hak Sung Lee², \\ In Guk Hwang ${ }^{3}$, Gu Seob Roh ${ }^{1}$, Hyun Joon Kim ${ }^{1}$, Gyeong Jae Cho' ${ }^{1}$, Wan Sung Choi ${ }^{1}$, \\ Dong Hoon Lee ${ }^{1}$ and ${ }^{\dagger}$ Sang Soo Kang ${ }^{1}$ \\ ${ }^{I}$ Dept. of Anatomy \& Convergence Medical Science, College of Medicine, Gyeongsang National University, \\ Jinju 52727, Korea \\ ${ }^{2}$ Nutraceutical Food R\&D Center, KolmarBNH Co., Ltd., Sejong 30003, Korea \\ ${ }^{3}$ Dept. of Agrofood Resources, National Academy of Agricultural Science, Rural Development Administration, \\ Wanju 55365, Korea
}

\begin{abstract}
Present study aimed to determine the effect of 'bitter melon', a popularly used fruit in Bangladesh and several other Asian countries, on high-fat-diet-induced type 2 diabetes. To investigate the effect, ethanol extract from bitter melon (BME) as a dietary supplement with mouse chow was used. BME was found to significantly attenuate the high-fat diet (HFD) -induced body weight and total fat mass. BME also effectively reduced the insulin resistance induced by the HFD. Furthermore, dietary supplementation of BME was highly effective in increasing insulin sensitivity and reducing hepatic fat and obesity. These results indicate that BME could be effective in attenuating type 2 diabetes and could therefore be a preventive measure against type 2 diabetes.
\end{abstract}

Key words : Bitter melon, Ethanol extract, High-fat diet, Type 2 diabetes, Sirtuin 1, Obesity

\section{INTRODUCTION}

Metabolic disorders such as obesity and type 2 diabetes (T2DM) are leading causes of morbidity and mortality for the world's growing population (Rodgers et al., 2005; Deshpande et al., 2008; Pfluger et al., 2008). In particular, T2DM is the most common, accounting for $90 \%$ of patients in these categories (Wang et al., 2017). Therefore, the effective control of blood glucose is the key to preventing adverse impacts on the quality of life for type 2 diabetes patients (Shafiee et al., 2012). T2DM is caused by resistance to the action of insulin combined with a deficiency in insulin secretion; recently, functional foods and their bioactive compounds have been found to be useful as complementary treatments for T2DM (Patel et al., 2012).

Bitter melon (Momordica charantia L.; MC), or bitter gourd, is a popular plant used for its anti-diabetic, anticancer, anti-inflammatory and cholesterol-lowering effects among the indigenous populations of Asia, South America, India, the Caribbean and East Africa. In Chinese and Indian traditional medicine, bitter melon has typically been used to treat hypoglycemia and diabetes (Tan et al., 2008).

\footnotetext{
Manuscript received September 11, 2017, Received in revised form September 15, 2017, Accepted September 17, 2017

$\dagger$ Corresponding Author : Sang Soo Kang, Dept. of Anatomy \& Convergence Medical Science, College of Medicine, Gyeongsang National University, 15 Jinjudaero 816 Beongil, Jinju, Gyeongnam 52727, Korea. Tel: +82-55-772-8030, Fax: +82-55-772-8039, E-mail: kangss@gnu.ac.kr

This is an Open Access article distributed under the terms of the Creative Commons Attribution Non-Commercial License (http:// creativecommons.org/licenses/by-nc/3.0) which permits unrestricted non-commercial use, distribution, and reproduction in any medium, provided the original work is properly cited.
} 
In previous studies, this plant was reported to have a preventive effect on the development of hyperglycemia in KK-Ay mice, which spontaneously develop T2DM (Alam et al., 2015). It was expected from these results that bitter melon would exhibit anti-diabetic effects in other diabetic animal models with insulin resistance (Efird et al., 2014). The physiologically active substance of bitter melon has been reported to be optimized by ethanol extraction (Fulco \& Sartorelli, 2008).

Recent epidemiological studies have indicated that the consumption of a high-calorie diet and the ensuing obesity are two of the principal causes of T2DM (Steyn et al., 2004). Therefore, an obesity-induced experimental model of T2DM induced by a high-calorie diet would be useful for evaluating the anti-diabetic action of bitter melon extract (Alam et al., 2015). C57BL/6 mice fed a high-fat diet (HFD) have been shown to be a representative model of obesity-induced diabetes (Hu \& Malik, 2010). The HFD causes insulin resistance, and insulin sensitivity is associated with the expression of Sirtuin 1 (SIRT1) (Mayoral et al., 2015). The SIRT1 pathway regulates energy homeostasis at the cellular and whole-body level. It plays a central role in the regulation of glucose uptake and insulin sensitivity (Li, 2013).

Many studies have reported that the expression of SIRT1 plays a role in glucose metabolism (de Kreutzenberg et al., 2010; Li \& Kazgan, 2011; Kitada \& Koya, 2013). In the present study, we found that ethanol extract of bitter melon has anti-obesity and anti-diabetic effects through sirt1 in the liver tissue. Therefore, we believe that the physiologically active substance of bitter melon can be used as an anti-diabetic agent to improve glucose metabolism by SIRT1.

\section{MATERIALS AND METHODS}

\section{Preparation of ethanol extract of bitter melon (Momordica charantia L.; MC)}

Three batches (No. DHP20160217-20160219) of stan- dardized ethanol extract of bitter melon (BME) compound were manufactured and verified by Daeho Corporation Co., Ltd. (Hwaseong, Korea). Briefly, the dried unripe fruit of bitter melon (Hamyang, Korea/Cheonryung Foods Co., Ltd.) was shattered and extracted by heating twice at $70^{\circ} \mathrm{C}$ (4 h and $2 \mathrm{~h}$ ) using 70\% ethanol. The extract was then filtered and concentrated (Busung Tech, Ansung, Korea) to 15-20 degrees Brix at $65^{\circ} \mathrm{C}$. The concentrated extract was spray dried (Mehyun Engineering, Anyang, Korea) at inlet temperature $175 \pm 10^{\circ} \mathrm{C}$ and outlet temperature $80 \pm 5^{\circ} \mathrm{C}$, and dextrin (49\%) and lecithin (1\%) were added. The extraction yield was approximately $34 \%(\mathrm{w} / \mathrm{w})$.

\section{High-fat-diet (HFD)-induced diabetic mice}

Five-week-old male C57BL/6J mice were purchased from Central Lab Animal Inc. (Seoul, Republic of Korea). After an adaptation period of 1 week, the mice were randomly divided into four groups $(n=8-12)$. The normal diet group (ND) was fed a chow diet $(10 \% \mathrm{kcal}$ from fat, New Brunswick, NJ, USA, Research Diets; D12450B) or a high-fat diet (HFD) (45\% kcal from fat, New Brunswick, NJ, USA, Research Diets; D12451) for 12 weeks and then was fed an HFD supplemented with BME 250 (250 mg/kg body weight/ day) or an HFD supplemented with BME $500(500 \mathrm{mg} / \mathrm{kg}$ body weight/day) for 12 weeks. The animals were housed in a temperature-controlled environment with a 12-h light /dark cycle during the entire 12-week experimental period.

The animals' food intake and body weight were measured weekly. At the end of the experimental period, the animals were sacrificed. Blood samples were collected from the heart using a 1-mL syringe and were drawn into a 5-mL VACUETTE tube (Greiner Bio-one, Thailand). After being centrifuged at $1,500 \times \mathrm{g}$ for $10 \mathrm{~min}$, the serum was stored at $-80^{\circ} \mathrm{C}$ until analysis. The liver was removed and weighed. Portions of mesenteric liver were immersed in $4 \%$ neutralized buffered paraformaldehyde (NBP) for histological inspection; other portions were frozen immediately in liquid nitrogen and stored at $-80^{\circ} \mathrm{C}$ for RNA and protein 
samples. The experimental protocol was approved by the Institutional Animal Care and Use Committee of Gyeongsang National University (Jinju, Republic of Korea).

\section{Hematoxylin and eosin (H\&E) staining}

Fixed liver tissue in 4\% neutral paraformaldehyde was dehydrated though a graded series of ethanol $(70 \%, 80 \%$, $90 \%, 95 \%$, and $100 \%$ absolute ethanol) for $2 \mathrm{~h}$ per concentration, cleared in two changes of xylene for $2 \mathrm{~h}$ per change and impregnated using two changes of molten paraffin wax for $2 \mathrm{~h}$ per change in a Leica tissue processor (Leica TP 1020, Germany). The embedded tissue was sectioned at a thickness of $5 \mu \mathrm{m}$ with a microtome (Leica RM2235, Germany) and subjected to H\&E staining. All the stained tissue sections were imaged with a virtual slide microscope (VS120 S1, Olympus BX61 VST, Hamburg, Germany) at $\times 20$ magnification with Olyvia software.

\section{Oil red O staining}

Fixed liver tissue in $4 \%$ neutral paraformaldehyde was cryoprotected in 30\% sucrose and embedded in Tissue-Tek optimal cutting temperature (OCT) compound (Sakura Fineteck USA, Inc., Torrance, CA, USA). The embedded tissue was cryosectioned at a thickness of 5 $\mu \mathrm{m}$ using a cryostat (Leica CM 1950, Germany) and subjected to oil red O staining with an Oil Red O kit (Abcam \#ab 150678). All the stained tissue sections were imaged with a virtual slide microscope (VS120 S1, Olympus BX61 VST, Hamburg, Germany) at $\times 20$ magnification with Olyvia software.

\section{Analysis of serum}

Serum samples were used to measure the content of free fatty acid (FFA), glucose, insulin, C-peptide, total cholesterol (TC) and low-density lipoprotein-cholesterol (LDLC). These analyses were carried out at the Green Cross Reference Laboratory (Seoul, Republic of Korea). C-peptide was measured with the Lbis (R) Mouse C-peptide
ELISA kit (U type) (Shibayagi Co., Ltd., Japan).

\section{Intraperitoneal glucose tolerance and in insulin} tolerance

The ND was fed a chow diet or a HFD for 12 weeks and then was fed an HFD supplemented with BME 250 or an HFD supplemented with BME 500 for 12 weeks. The performed glucose tolerance tests with HFD, BME 250 and 500 mice before and after transplantation after $16 \mathrm{~h}$ of fasting by measuring glycemic values in peripheral blood following intraperitoneal (i.p.) injection of $4 \mathrm{~g} / \mathrm{kg}$ body weight glucose in saline. Intraperitoneal glucose tolerance test (IPGTT) under stress conditions was performed by keeping the animals inside restraining boxes and injecting i.p. $2 \mathrm{~g} / \mathrm{kg}$ glucose in saline. Intraperitoneal insulin tolerance tests (IPITT) were performed on random-fed mice after i.p. injection of $0.75 \mathrm{U} / \mathrm{kg}$ insulin in saline.

\section{Western blot and protein quantification}

Tissues were lysed RIPA buffer (Thermo Fisher Scientific, Waltham, MA, USA). Total protein concentrations were determined using BCA assay (PIERCE, Rockford, IL, USA). An equal volume of $4 \times$ SDS sample buffer was added, and the samples were boiled for $5 \mathrm{~min}$. Equivalent amounts of total protein $(20-60 \mu \mathrm{g})$ were separated by SDS-PAGE on $8-12 \%$ polyacrylamide gel and then transferred to nitrocellulose membrane. The membrane was blocked with 5\% skim milk or $3 \%$ BSA in $0.1 \%$ Tween-20/Tris buffered saline (TBS-T). The membrane was incubated with SirT1 (1:1000, \#2493, Cell Signaling Technology, MA), PPAR $\alpha$ (1:1000, Santa Cruz Biotechnology, CA), SREBP-1(1: 1000, sc-8984, Santa Cruz Biotechnology), AMPK (1: 1000, \#2532, Cell Signaling Technology), p-AMPK (1: 1000, \#2535, Cell Signaling Technology), ACC (1:1000, \#3676, Cell Signaling Technology), p-ACC (1:1000, \#11818, Cell Signaling Technology), $\alpha$-tublin (1:10000, A11126, Thermo Fisher Scientific) and $\beta$-actin (1:10000, MA5-15739, Thermo Fisher Scientific ) antibodies overnight at $4{ }^{\circ} \mathrm{C}$. 


\section{Statistical analysis}

The data are expressed as the mean and standard error of the mean (means \pm SEM). The differences among the groups were analyzed by one-way analyses of variance (ANOVA), followed by Tukey's HSD test in the GraphPad Prism 5 software (GraphPad Software Inc., La Jolla, CA).

\section{RESULTS}

\section{Effects of BME on body weight gain and organ} weight

The C57BL/6 mice were randomly divided into four groups $(n=8-12)$. The first group was fed a normal diet (ND), the second was fed a HFD, and the other two groups were fed an HFD supplemented with $250 \mathrm{mg} \mathrm{BME} / \mathrm{kg} \mathrm{BW}$ (BME 250) and $500 \mathrm{mg} \mathrm{BME} / \mathrm{kg}$ BW (BME 500), respectively. The body-weight gain in the HFD group was significantly greater than that in the ND group. Notably, the body-weight gain in both the BME 250 and the BME 500 group was significantly lower than that in the HFD group (Fig. 1A). Whereas food intake in both the BME 250 and the BME 500 group was not significantly different than that in the HFD group (Fig. 1B). The weight of individual or total white adipose tissue (total WAT, epididymal, perirenal, and mesenteric) was significantly greater in the HFD group than in the ND group. The WAT weight decreased significantly after the administration of BME (Fig. 1C). BME inhibited visceral fat accumulation without affecting food intake. Liver weight decreased significantly more in the BME 250 and the BME 500 group than in the HFD group (Fig. 1D). The H\&E and oil red O staining showed that BME ameliorated hepatic steatosis more in the BME 500 group than in the HFD group (Fig. 1E).

\section{Effect of BME on plasma lipids}

The plasma lipid (total cholesterol, LDL cholesterol, FFA) levels in the serum decreased significantly in the BME 250 and 500 groups compared with the HFD group

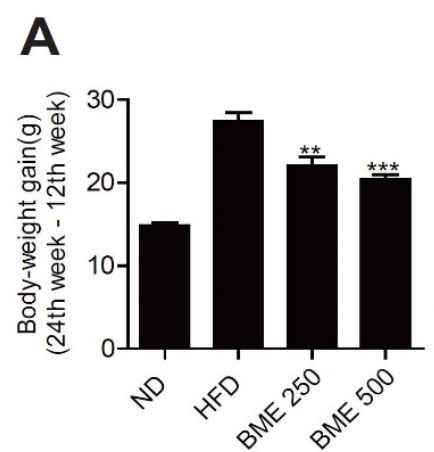

B

C

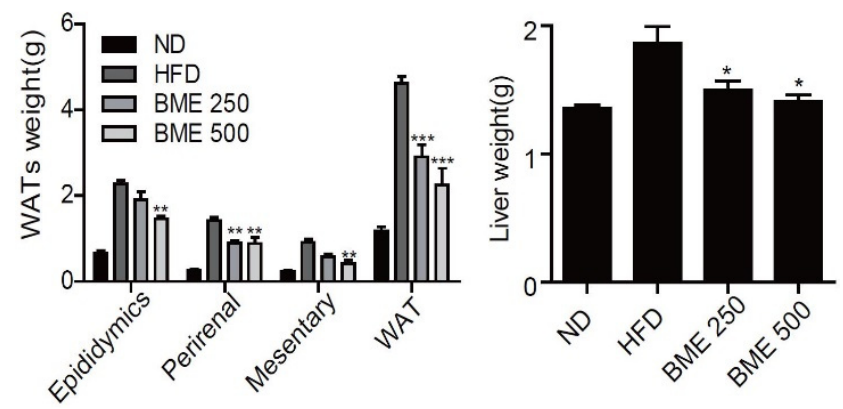

E
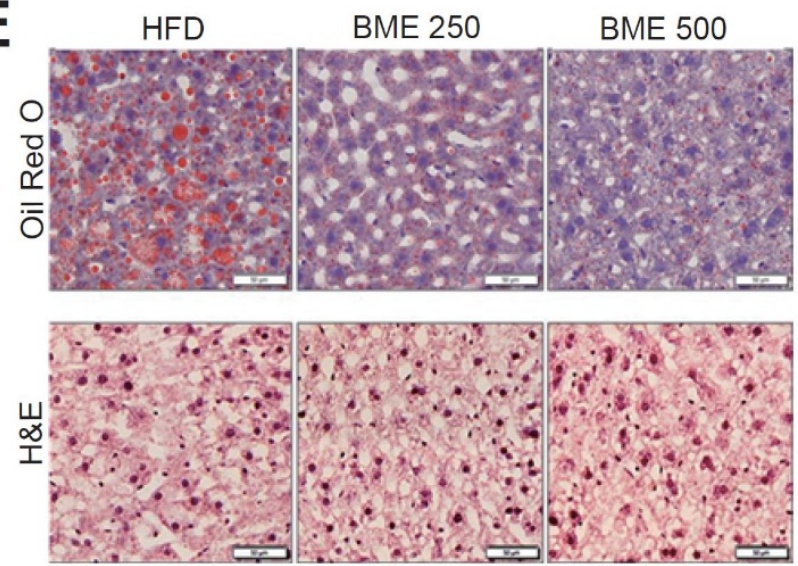

Fig. 1. Effects of BME on body weight gain, food intake, and organ weight. (A) body weight gain, (B) food intake rate, (C) WAT weight,(D) liver weight, (E) liver stained with Oil red O. ND, normal-fat diet; HFD, high-fat diet: BME 250, HFD plus $250 \mathrm{mg} /$ $\mathrm{kg}$ of ethanol extract powder of bitter melon; BME 500 , HFD plus $500 \mathrm{mg} / \mathrm{kg}$ of ethanol extract powder of bitter melon. Values are expressed as a mean \pm SEM (n=8). ${ }^{*} p<0.05,{ }^{* *} p<0.01{ }^{* * *} p<0.005$ vs. the HFD group.

(Fig. 2 A-C). As lipolysis, circulating free fatty acids under insulin resistance conditions, these results demonstrate 

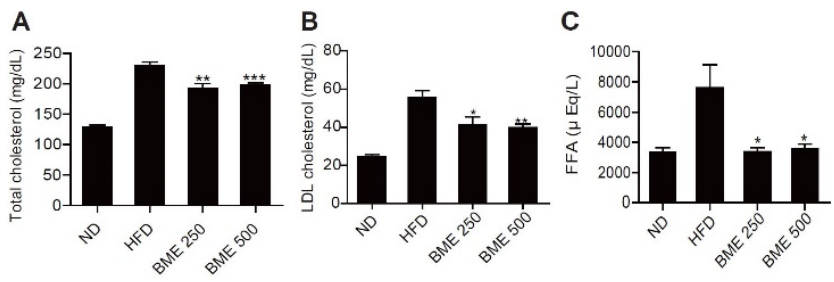

Fig. 2. Effects of BME on serum levels of lipid. (A) plasma total cholesterol levels, (B) plasma LDL cholesterol levels, (C) plasma FFA cholesterol levels. ND, normal-fat diet; HFD, high-fat diet: BME 250, HFD plus $250 \mathrm{mg} / \mathrm{kg}$ of ethanol extract powder of bitter melon; BME 500, HFD plus $500 \mathrm{mg} / \mathrm{kg}$ of ethanol extract powder of bitter melon. Values are expressed as a mean \pm SEM $(\mathrm{n}=8) .{ }^{*} p<0.05,{ }^{* *} p<$ $0.01^{* * *} p<0.005$ vs. the HFD group.

that the decrease in plasma lipids may contribute at least partially to the improvement of diabetes.

\section{Effects of BME on glucose intolerance}

The effects of BME supplementation on fasting blood glucose levels decreased significantly more in the BME 250 and 500 groups than in the HFD group (Fig. 3A), and the plasma insulin and C-peptide levels decreased more in the BME 500 group than in the HFD group (Fig. 3 B-C).

An IPGTT performed to determine the effect of BME on glucose tolerance data showed that the glucose concentration was significantly lower in the BME 200 and BME 500 groups than in the HFD group at 30, 60 and 120 min postglucose i.p. injection (Fig. 3D). Glucose disappearance, based on IPITT results, demonstrated that BME significantly improved glucose disposal ability by enhancing insulin sensitivity (Fig. 3E).

\section{BME activation of the SIRT1 signaling pathway} in the liver

To elucidate which genes are involved in the antisteatotic and anti-diabetic effect of BME, the expressions of the genes related to hepatic lipid metabolism were measured by quantitative Western blot. The HFD group had a significantly lower expression of SIRT1 than the BME 250
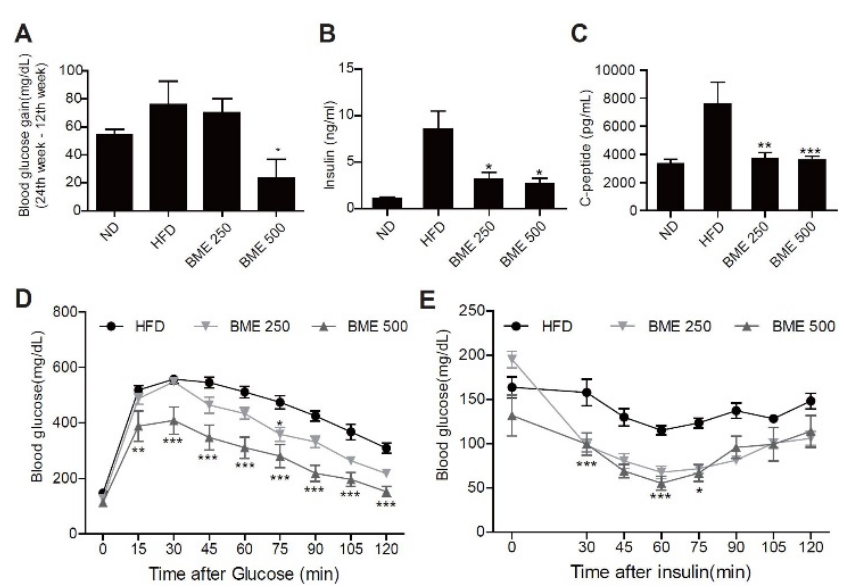

Fig. 3. Effects of BME on the plasma biochemical parameters. (A) plasma fasting glucose levels, (B) plasma insulin levels, (C) plasma C-peptide levels, (D) IPITT, (E) IPGTT. ND, normal-fat diet; HFD, High-fat diet; BME 250, HFD plus $250 \mathrm{mg} / \mathrm{kg}$ of ethanol extract powder of bitter melon; BME 500, HFD plus $500 \mathrm{mg} / \mathrm{kg}$ of ethanol extract powder of bitter melon. Values are expressed as a mean \pm SEM (n=8). ${ }^{*} p<0.05,{ }^{* *} p<0.01{ }^{* * *} p<0.005$ vs. the HFD group.

and 500 groups. The BME groups showed a significant increase in the hepatic expression levels of SIRT1 (Fig. 4A). Similarly, the expression of phospho-AMPK (p-AMPK), p-ACC and ACC increased significantly in the BME 500 group (Fig. 4B). The down-signaling molecules of SIRT1, such as Foxol and PPAR $\alpha$ were increased significantly and SREBP1c was decreased significant in the BME 250 and 500 group (Fig. 4C).

\section{DISCUSSION}

In the present study, we demonstrated that the effect of bitter melon extract on the HFD-induced obesity and T2DM in C57BL/6 mice. Already numerous people use bitter melon for the prevention and primary therapeutics for the early T2DM like status. This study aimed to evaluate the precise effects of bitter melon on the lipid accumulation and development of T2DM. We used only premature bitter melon (before the formation of seed) to avoid the toxicity. 
A

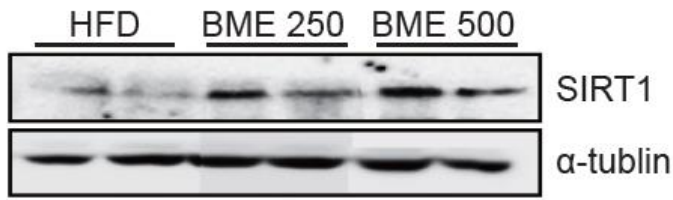

B

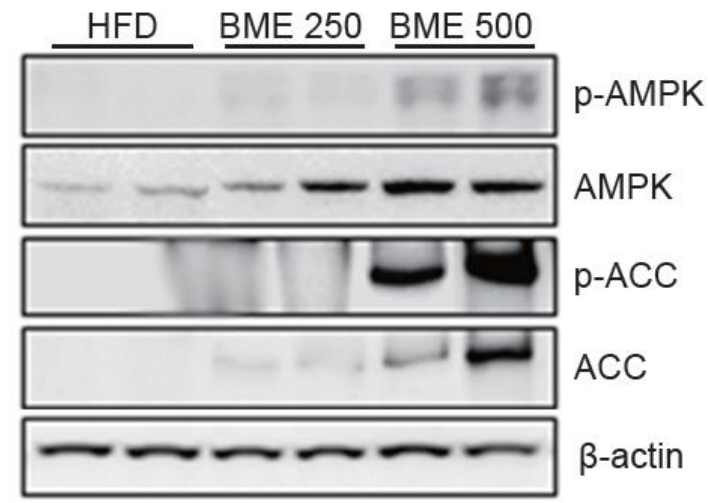

C

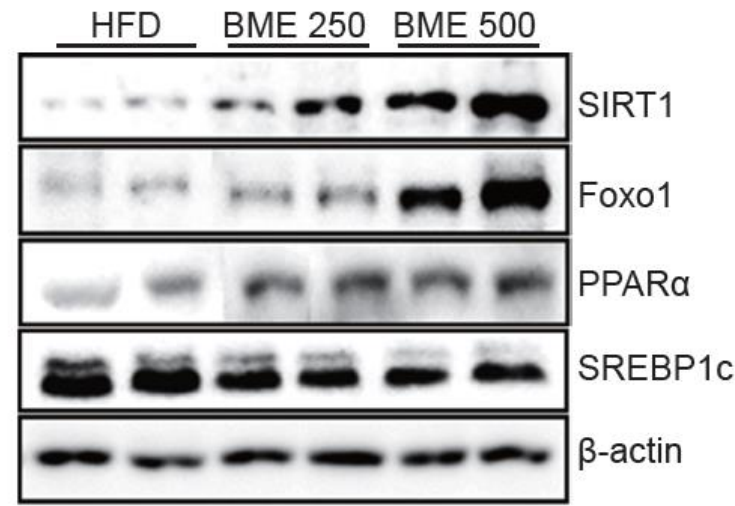

Fig. 4. Effects of BME on hepatic metabolic signaling pathway. (A) protein expressions of liver SIRT1 (B), phosphorylated AMPK, ACC (C), SIRT1, Foxo1, PPAR $\alpha$ and SREBP1c were determined by Western blotting. HFD was used as a control and $\beta$-actin, $\alpha$ tubulin ware used as a loading control.

One of the hallmarks of the development of metabolic syndrome and T2DM is the elevation of ectopic lipid accumulation in non-subcutaneous adipose stores (increased central adiposity, muscle and liver lipid accumulation) (Lara-Castro \& Garvey, 2008). BME inhibited visceral fat accumulation without affecting food intake. Therefore, it is suggested that an adequate administration of BME might effectively reduce hepatic steatosis. Actually, increased visceral adiposity is characterized as an important factor in the development of T2DM in human subjects (Bray et al.,
2008; Ginsberg \& MacCallum, 2009). Numerous studies have demonstrated the link between ectopic fat deposition and insulin resistance in persons with overt T2DM (Unger, 2003; Lara-Castro \& Garvey, 2008). Momordica charantia is a food source that contains various bioactive compounds with health benefits (Ruda-Kucerova et al., 2015). Previous studies have shown that in mice fed HFD, freeze-dried Momordica charantia juice was found to possess the potential to reduce adiposity, and extracts from this plant may reduce visceral obesity (Chen et al., 2003; Alam et al., 2015; Mahmoud et al., 2017).

In present study, we investigated whether BME could improve HFD-induced obesity in C57BL/6 mice and explored the mechanisms. C57BL/6 mice were maintained on an ND or HFD for 12 weeks and then fed the ND, HFD, BME 250, or BME 500 for another 12 weeks. The weekly food intakes among the groups did not differ from each other. BME obviously reduced the weight of WAT and the liver in the HFD group, which was consistent with the results of changes in body weight. These results suggested that the BME-mediated decrease in body weight was attributable to a reduction in the weight of WAT and the liver independent of food intake.

It has been reported that hyperglycemia is the defined pathological feature of T2DM. Effective control of glucose levels is crucial for preventing diabetic complications and improving patients' quality of life (Shafiee et al., 2012; Agrawal, 2016). Present results of IPGGT and IPITT suggested that BME may improve T2DM pathologies by alleviating hyperglycemia.

SIRT1 mediates metabolic benefits in various tissues. In the liver, SIRT1 supports gluconeogenesis via peroxisome proliferator-activated receptor (PPAR a ) and forkhead box O1 (FOXO1) (Rodgers et al., 2005) and inhibits fatty acid synthesis by targeting sterol regulatory element binding protein 1c (SREBP1c) for degradation (Walker et al., 2010). The down-signaling molecules of SIRT1, such as Foxo1 and PPAR $\alpha$ were increased significantly and SREBP1c 
was decreased significant in the BME administrated animals. These findings showed that BME increased hepatic gluconeogenesis and fatty acid use by up-regulating a SIRT1 signaling pathway. As also, BME up-regulated the expression levels of p-AMPK and p-ACC. These data showed that on one hand, BME may up-regulate SIRT1 to induce gluconeogenesis; on the other hand, it may stimulate AMPK and ACC to promote fatty acid oxidation in the liver. Recent studies have shown that aqueous extract of Momordica charantia seeds regulated the insulin signaling pathway in muscles and adipose tissues primarily by targeting insulin receptors (IR) (Lo et al., 2013). Here, for the first time, we illustrated that BME reinforce HFD-induced gluconeogenesis and fatty acid use in the livers of mice by modulating SIRT1 signaling. SIRT1 is involved in protection against metabolic syndrome. However, SIRT1's purpose in protecting against metabolic syndrome is known mostly through indirect evidence by using the chemical activators of SIRT1(Pfluger et al., 2008). Studies have demonstrated the beneficial effects of bitter melon extract on HFD-induced obesity, including the improvement of insulin sensitivity and hepatic steatosis by regulating the SIRT1 signaling pathway. Similarly, in this study, BME not only enhanced the expression levels of SIRT1 but also up-regulated Foxo1 and PPAR $\alpha$, as also down-regulated SREBP1c. In fact, the underlying mechanisms of hepatic metabolism in these animal models are different; it seems that BME may act on a particular pathway, depending on the cause of T2DM development. In this study, BME increased the phosphorylation levels of AMPK in the liver. Briefly, the stimulation of AMPK in the liver may be the consequence of a reduction in the AMPK/ACC pathway, not the SIRT1/ AMPK pathway.

In conclusion, BME not only attenuated HFD-induced hepatic steatosis but also ameliorated HFD-induced insulin resistance by improving insulin sensitivity. The reduced circulatory insulin inhibited expressions of lipogenic genes in the liver and attenuated hepatic steatosis.
In summary, BME greatly enhanced insulin sensitivity, reversed liver steatosis, reduced body-fat mass and increased the expression level of SIRT1 in mice with HFDinduced T2DM. This study suggested that the favorable effects of BME on increasing insulin sensitivity and attenuating hepatic steatosis may be attributed to the mediation of SIRT1 and AMPK-ACC signaling. Therefore, BME can be viewed as a potential natural resource for the development of new therapeutic alternatives in the treatment of obesity and T2DM.

\section{ACKNOWLEDGMENTS}

This work was supported by the Cooperative Research Program for Agriculture Science and Technology Development (project no. PJ00010003022016) of the Rural Development Administration, Republic of Korea.

\section{DISCLOSURE}

The authors declare no conflict of interest.

\section{REFERENCES}

Agrawal S (2016) Role of self- Care in management of diabetes mellitus. J Assoc Physicians India 64:92.

Alam MA, Uddin R, Subhan N, Rahman MM, Jain P, Reza HM (2015) Beneficial role of bitter melon supplementation in obesity and related complications in metabolic syndrome. J Lipids 2015: 496169.

Bray GA, Jablonski KA, Fujimoto WY, Barrett-Connor E, Haffner S, Hanson RL, Hill JO, Hubbard V, Kriska A, Stamm E, Pi-Sunyer FX (2008) Relation of central adiposity and body mass index to the development of diabetes in the diabetes prevention program. Am J Clin Nutr 87:1212-1218.

Chen Q, Chan LL, Li ET (2003) Bitter melon (Momordica charantia) reduces adiposity, lowers serum insulin and 
normalizes glucose tolerance in rats fed a high fat diet. J Nutr 133:1088-1093.

De Kreutzenberg SV, Ceolotto G, Papparella I, Bortoluzzi A, Semplicini A, Dalla Man C, Cobelli C, Fadini GP, Avogaro A (2010) Downregulation of the longevityassociated protein sirtuin 1 in insulin resistance and metabolic syndrome: potential biochemical mechanisms. Diabetes 59:1006-1015.

Deshpande AD, Harris-Hayes M, Schootman M (2008) Epidemiology of diabetes and diabetes-related complications. Phys Ther 88:1254-1264.

Efird JT, Choi YM, Davies SW, Mehra S, Anderson EJ, Katunga LA (2014) Potential for improved glycemic control with dietary Momordica charantia in patients with insulin resistance and pre-diabetes. Int $\mathrm{J}$ Environ Res Public Health 11: 2328-2345.

Fulco M, Sartorelli V (2008) Comparing and contrasting the roles of AMPK and SIRT1 in metabolic tissues. Cell Cycle 7: 3669-3679.

Ginsberg HN, Maccallum PR (2009) The obesity, metabolic syndrome, and type 2 diabetes mellitus pandemic: Part I. Increased cardiovascular disease risk and the importance of atherogenic dyslipidemia in persons with the metabolic syndrome and type 2 diabetes mellitus. J Cardiometab Syndr 4:113-119.

Hu FB, Malik VS (2010) Sugar-sweetened beverages and risk of obesity and type 2 diabetes: epidemiologic evidence. Physiol Behav 100: 47-54.

Kitada M, Koya D (2013) SIRT1 in type 2 diabetes: mechanisms and therapeutic potential. Diabetes Metab J 37: 315-325.

Lara-Castro C, Garvey WT (2008) Intracellular lipid accumulation in liver and muscle and the insulin resistance syndrome. Endocrinol Metab Clin North Am 37:841-856.

Li X (2013) SIRT1 and energy metabolism. Acta Biochim Biophys Sin (Shanghai) 45:51-60.

Li X, Kazgan N (2011) Mammalian sirtuins and energy metabolism. Int J Biol Sci 7:575-587.

Lo HY, Ho TY, Lin C, Li CC, Hsiang CY (2013) Momordica charantia and its novel polypeptide regulate glucose homeostasis in mice via binding to insulin receptor. J Agric Food Chem 61:2461-2468.

Mahmoud MF, El Ashry FE, El Maraghy NN, Fahmy A (2017) Studies on the antidiabetic activities of Momordica charantia fruit juice in streptozotocin-induced diabetic rats. Pharm Biol 55:758-765.

Mayoral R, Osborn O, Mcnelis J, Johnson AM, Oh DY, Izquierdo CL, Chung H, Li P, Traves PG, Bandyopadhyay G, Pessentheiner AR, Ofrecio JM, Cook JR, Qiang L, Accili D, Olefsky JM (2015) Adipocyte SIRT1 knockout promotes PPARgamma activity, adipogenesis and insulin sensitivity in chronic-HFD and obesity. Mol Metab 4:378-391.

Patel DK, Prasad SK, Kumar R, Hemalatha S (2012) An overview on antidiabetic medicinal plants having insulin mimetic property. Asian Pac J Trop Biomed 2:320330.

Pfluger PT, Herranz D, Velasco-Miguel S, Serrano M, Tschop MH (2008) Sirt1 protects against high-fat dietinduced metabolic damage. Proc Natl Acad Sci USA 105:9793-9798.

Rodgers JT, Lerin C, Haas W, Gygi SP, Spiegelman BM, Puigserver P (2005) Nutrient control of glucose homeostasis through a complex of PGC-1alpha and SIRT1. Nature 434:113-118.

Ruda-Kucerova J, Kotolova H, Koupy D (2015) Effectiveness of phytotherapy in supportive treatment of type 2 diabetes mellitus III. Momordica (Momordica charantia). Ceska Slov Farm 64:126-132.

Shafiee G, Mohajeri-Tehrani M, Pajouhi M, Larijani B (2012) The importance of hypoglycemia in diabetic patients. J Diabetes Metab Disord 11:17.

Steyn NP, Mann J, Bennett PH, Temple N, Zimmet P, Tuomilehto J, Lindstrom J, Louheranta A (2004) Diet, nutrition and the prevention of type 2 diabetes. Public 
Health Nutr 7:147-165.

Tan MJ, Ye JM, Turner N, Hohnen-Behrens C, Ke CQ, Tang CP, Chen T, Weiss HC, Gesing ER, Rowland A, James DE, Ye Y (2008) Antidiabetic activities of triterpenoids isolated from bitter melon associated with activation of the AMPK pathway. Chem Biol 15:263-273.

Unger RH (2003) Minireview: weapons of lean body mass destruction: the role of ectopic lipids in the metabolic syndrome. Endocrinology 144:5159-5165.

Walker AK, Yang F, Jiang K, Ji JY, Watts JL, Purushotham A, Boss O, Hirsch ML, Ribich S, Smith JJ, Israelian K,
Westphal CH, Rodgers JT, Shioda T, Elson SL, Mulligan P, Najafi-Shoushtari H, Black JC, Thakur JK, Kadyk LC, Whetstine JR, Mostoslavsky R, Puigserver P, Li X, Dyson NJ, Hart AC, Naar AM (2010) Conserved role of SIRT1 orthologs in fasting-dependent inhibition of the lipid/cholesterol regulator SREBP. Genes Dev 24:1403-1417.

Wang G, Zhang Z, Feng Y, Sun L, Xiao X, Wang G, Gao Y, Wang H, Zhang H, Deng Y, Sun C (2017) Telemedicine in the management of type 2 diabetes mellitus. Am J Med Sci 353:1-5. 